\title{
Risk Factors Associated with Methicillin-resistant Staphylococcus aureus Infection in Children
}

\author{
Kandasamy Senthilkumar, Niranjan Biswal AND *Sujatha Sistla \\ From Departments of Pediatrics and *Microbiology, Jawaharlal Institute of Postgraduate Medical Education and Research \\ (JIPMER), Pondicherry, India.
}

Correspondence:

Dr Niranjan Biswal, Professor, Department of Pediatrics, JIPMER, Pondicherry-605006, India. drnbiswal@yahoo.com Received: April 22, 2014; Initial review: June 10, 2014; Accepted: November 10, 2014
Objective: To identify the clinical variables that differentiate MRSA (Methicillin-resistant Staphylococcus aureus) from MSSA (Methicillin-sensitive S. aureus) infection. Methods: Cases having culture isolates of Staphylococcus species were recruited. Baseline and other laboratory parameters were compared between MSSA and MRSA sub-groups to identify the predictors for MRSA. Results: Out of 98 isolates of S.aureus, $46(47 \%)$ were MRSA. Significant leukocytosis was found in cases with MRSA $(P<0.03)$. None of the other clinical variables could differentiate MRSA from MSSA infection. Conclusion: Presence of leukocytosis was twice more likely to predict MRSA than MSSA at admission. Empiric therapy must be guided by antimicrobial sensitivity pattern of regional culture isolates.

Keywords: MRSA, Risk factors.
M ethicillin-resistant Staphylococcus aureus (MRSA) is a common multidrug resistant pathogen worldwide [1]. Emergence of community-associated MRSA (CAMRSA) infections has a fundamentally different epidemiology compared to that of hospital-associated MRSA (HA-MRSA) infections [2]. CA-MRSA infections often do not have previous health care exposure and commonly manifests as skin and soft-tissue infections [3]. There is a paucity of data on childhood MRSA infection in developing countries. Knowledge on its prevalence and risk factors may help to plan the preventive strategies.

\section{MethodS}

This study was done in the Department of Pediatrics and Microbiology, JIPMER, Pondicherry, India (August 2011 to July 2013) after obtaining clearance from the Institute Ethics Committee.

The primary objective was to identify clinical variables that could differentiate MRSA and MSSA (Methicillin-sensitive Staphylococcus aureus) infection in children (0-12 years) treated with confirmed Staphylococcus infections. Isolates were identified as S.aureus using standard microbiological methodology from blood, pus, urine, CSF (cerebrospinal fluid) and pleural fluid. Oxacillin screen agar was used to detect methicillin resistance in Mueller Hinton Agar supplemented with $6 \mu \mathrm{g} / \mathrm{mL}$ of Oxacillin sodium and $4 \% \mathrm{NaCl}$ as per Clinical and Laboratory Standards Institute recommendations [4]. Control strains used in this study were ATCC 29213 for MSSA and ATCC 43300 for MRSA.

Accompanying Editorial: Pages 22-24.

Informed consent was taken from the parents. Baseline demographic data was recorded. Details of prior hospitalization and antibiotic usage within 12 months, history of exanthematous illness like measles and varicella, abrasion/laceration, contact with potential S.aureus carrier, malnutrition, and immunosuppressant usage were recorded. Specimens were collected at admission from all the patients except from those with hospital-acquired S.aureus infection. Children with confirmed S.aureus infection were classified into MSSA and MRSA subgroups. Cases with a previous history of hospitalization, septic shock, tricuspid valve endocarditis and pyogenic arthritis were suspected to have MRSA infection. MRSA was further classified into CA-MRSA and HA-MRSA subgroups. All the cases were followed up till discharge or death.

A case was classified as Hospital-onset if the MRSA culture was obtained on or after the fourth calendar day of hospitalization, where admission is hospital day 1; as healthcare-associated community-onset (HACO) if the culture was obtained in an outpatient setting or before the fourth calendar day of hospitalization and had one of more of the following: (i) a history of hospitalization, 
TABLE I COMPARISON OF Risk FACTORS IN AgE Group OF $>1$ MONTH* -12 YEARS

\begin{tabular}{|c|c|c|c|}
\hline Methici & $\begin{array}{l}\text { in-resistant S.aureus } \\
\qquad \begin{array}{l}n=35 \\
\mathrm{No}(\%)\end{array}\end{array}$ & $\begin{array}{c}\text { Methicillin-sensitive S.aureus } \\
n=43 \\
\text { No(\%) }\end{array}$ & $\begin{array}{c}\text { OR } \\
(95 \% C I)\end{array}$ \\
\hline Hospitalization in prior $12 \mathrm{mo}$ & $6(17)$ & $5(12)$ & $1.57(0.43-5.66)$ \\
\hline Exanthematous illness (fever with rash) in prior $12 \mathrm{mo}$ & $4(11)$ & $2(5)$ & $2.64(0.43-21.5)$ \\
\hline History of minor truma causing skin discontinuity & $5(14)$ & $6(14)$ & $1.03(0.28-3.69)$ \\
\hline Antibiotic usage in prior $12 \mathrm{mo}$ & $6(17)$ & $8(19)$ & $0.91(0.28-2.90)$ \\
\hline Immunosuppressant usage & $1(3)$ & $2(5)$ & $0.6(0.01-6.90)$ \\
\hline Contact with potential S. aureus infected patient & $0(0)$ & $2(5)$ & - \\
\hline
\end{tabular}

${ }^{*}$ Risk factors are not applicable in $\leq 1$ month; $P>0.05$ for all comparisons.

surgery, dialysis, or residence in a long term care facility in the previous year, or (ii) the presence of a central vascular catheter within 2 days prior to MRSA culture; and as Community-associated (CA), if none of the previously mentioned criteria were met [4].

Anemia was defined as reduction of the hemoglobin concentration below the range for age and sex [6]. Leukocytosis was defined as white blood cell (WBC) count $>2$ SD above the mean for age [7], and thrombocytopenia as platelet count $<1,50,000 / \mathrm{cu} \mathrm{mm}$ [8]. Sepsis: Systemic Inflammatory Response Syndrome (SIRS) associated with suspected or proven infection (by positive culture, tissue stain, or polymerase chain reaction test) as per International Pediatric Sepsis consensus conference guidelines [7].

\section{Results}

Out of 210 patients screened, 98 isolates were S.aureus. Out of 98 isolates of S.aureus, $52(53 \%)$ were MSSA. Thirty seven (80\%) isolates of MRSA were community acquired and $9(20 \%)$ were hospital acquired. MRSA occurrence among skin and soft tissue infections (SSTI), empyema, and bacteremia were $44 \%, 66 \%, 30 \%$, respectively. Demographic variables were comparable in both the groups. Associated risk factors for possible S.aureus infection could be identified in $49 \%$ of the study population (Table I). There was no significant difference in the number of children with PEM between MRSA and MSSA subgroup.

Proportion of cases with leukocytosis were significantly higher in the MRSA than MSSA $(\mathrm{P}<0.03)$ (Table II). Susceptibility of MRSA to vancomycin was $100 \%$ and to clindamycin was $63 \%$. Eight strains of MRSA and six strains of MSSA had inducible clindamycin resistance detected by D test. Clindamycin resistance in SSTIs was 25\%. Cases with MRSA infection stayed 4.8 days longer in hospital than the MSSA. Case fatality was similar in both the groups (MRSA $=7 \%$, MSSA $=4 \% ; P=0.883$ ).

\section{Discussion}

SSTI were the predominant sites for both MRSA (42\%) and MSSA (46\%) followed by blood stream infection, similar to another study from India [9]. Clinical variables were not different between MRSA and MSSA subgroups. None of the clinical variables could predict MRSA infection at admission like other studies [10,11].

Though nearly half of the cases with MSSA had leukocytosis, significant leucocytosis was most often associated with MRSA infection. Presence of leukocytosis in staphylococcal bone and joint infections was an excellent predictor of MRSA in two other studies $[12,13]$.

Following an uniform antibiotic policy of administering cloxacillin to all the suspected staphylococcal infection, antibiotic change to vancomycin was needed in $67 \%$ of cases who later grew MRSA. Among very sick children treated with

TABLE II COMPARISON OF LABORATORY VALUES AND CLINICAL COURSE During Hospital Stay

\begin{tabular}{|c|c|c|c|c|}
\hline Variables & $\begin{array}{c}M R S A \\
n=46\end{array}$ & $\begin{array}{c}M S S A \\
n=52\end{array}$ & $\begin{array}{c}\text { OR } \\
(95 \% C I)\end{array}$ & Pvalue \\
\hline Anaemia & $13(28)$ & $18(35)$ & $0.74(0.29-1.91)$ & 0.50 \\
\hline Leukocytosis & $35(76)$ & $29(56)$ & $2.52(1.06-6.30)$ & 0.04 \\
\hline Thrombocytopenia & $2(4)$ & $4(8)$ & $0.79(0.04-4.04)$ & 0.80 \\
\hline Septic shock & $6(13)$ & $7(14)$ & $0.96(0.26-3.55)$ & 0.95 \\
\hline Needed ICU stay & $5(11)$ & $7(14)$ & $0.78(0.20-3.05)$ & 0.70 \\
\hline $\begin{array}{l}\text { Requring mechani- } \\
\text { cal ventilation }\end{array}$ & $5(11)$ & $7(14)$ & $0.78(0.20-3.05)$ & 5) 0.70 \\
\hline
\end{tabular}




\section{What This Study Adds?}

- Clinical and epidemiologic characteristics cannot distinguish MRSA from MSSA infection.

- It is twice more likely to isolate MRSA rather than MSSA from a child when total leukocyte count is elevated (2SD for age).

vancomycin for suspected MRSA, switching over to cloxacillin was needed in $23 \%$ as they grew MSSA. Hence, severity of clinical condition at admission may not be a useful indicator to initiate empiric antibiotic therapy for MRSA.

Convenience sampling was used in this study. Culture isolation was performed at a single tertiary center; therefore, the findings may not be extrapolated to other areas where circulating strain types may differ. Multicentric studies with a larger population involving community may be needed to validate these findings. Differentiation between CA-MRSA and HA-MRSA was based on epidemiological factors alone and not on molecular analysis of SCC mecs, which is the gold standard.

Risk factors being similar in MRSA and MSSA infections, standard hygienic measures and proper treatment guidelines would be beneficial in controlling both MRSA and MSSA. Empiric antibiotics for suspected staphylococcal infection must be guided by frequently analyzing the hospital culture isolates and sensitivity pattern rather than relying on clinical variables and pointers in the history.

Contributors: KS and NB: were involved in designing the study and management of the patients; KS: collected the data and reviewed the literature. NB conceptualized the study, reviewed the literature and critically reviewed the manuscript; SS: provided guidance regarding laboratory evaluation and also critically reviewed the manuscript. All authors contributed to drafting of the manuscript and approved the final version of the manuscript.

Funding: None; Competing interest: None stated.

\section{REFERENCES}

1. Cosgrove SE, Fowler VG Jr. Management of methicillinresistant Staphylococcus aureus bacteremia. Clin Infect Dis. 2008;46:S386-93.

2. Boucher HW, Corey GR. Epidemiology of methicillinresistant Staphylococcus aureus. Clin Infect Dis. 2008;46:S344-9.

3. Stryjewski ME, Chambers HF. Skin and soft-tissue infections caused by community-acquired methicillinresistant Staphylococcus aureus. Clin Infect Dis. 2008;46:S368-77.

4. Clinical and Laboratory Standards Institute. 2013.
Performance standards for Antimicrobial susceptibility testing; twenty third Informational supplement. M100S23. Accessed September 24, 2014.

5. Centers for Disease Control and Prevention. 2011. Active Bacterial Core Surveillance Report, Emerging Infections Program Network, Methicillin-Resistant Staphylococcus aureus, 2011. Available from: http://www.cdc.gov/abcs/ reportsfindings/survreports/mrsa11. Accessed September 23, 2014.

6. Lerner Norma B. The anaemia. In: Kliegman RM, Stanton BF, St.Geme JW, Schor NF, Behrman RE. Nelson Textbook of Pediatrics. 19th ed. Philadelphia,: Elsevier; 2011.p.1648.

7. Goldstein B, Giroir B, Randolph A, International Consensus Conference on Pediatric Sepsis. International pediatric sepsis consensus conference: definitions for sepsis and organ dysfunction in pediatrics. Pediatr Crit Care Med. 2005;6:2-8.

8. Dellinger RP, Levy MM, Rhodes A, Annane D, Gerlach $\mathrm{H}$, Opal SM, et al. Surviving Sepsis Campaign: international guidelines for management of severe sepsis and septic shock, 2012. Intensive Care Med. 2013;39:165-228.

9. Joshi S, Ray P, Manchanda V, Bajaj J, Chitinis DS, Gautam V, et al. Methicillin resistant Staphylococcus aureus (MRSA) in India: Prevalence and susceptibility pattern. Indian J Med Res. 2013;137:363-9.

10. Golding GR, Levett PN, McDonald RR, Irvine J, Nsungu $\mathrm{M}$, Woods $\mathrm{S}$, et al. A comparison of risk factors associated with community-associated methicillin-resistant and susceptible Staphylococcus aureus infections in remote communities. Epidemiol Infect. 2010;138:730-7.

11. Miller LG, Perdreau-Remington F, Bayer AS, Diep B, Tan N, Bharadwa K, et al. Clinical and epidemiologic characteristics cannot distinguish community-associated methicillin-resistant Staphylococcus aureus infection from methicillin-susceptible S. aureus infection: a prospective investigation. Clin Infect Dis. 2007;44:47182.

12. Kini AR, Shetty V, Kumar AM, Shetty SM, Shetty A. Community-associated, methicillin-susceptible, and methicillin-resistant Staphylococcus aureus bone and joint infections in children: experience from India. J Pediatr Orthop Part B. 2013;22:158-66.

13. Ju KL, Zurakowski D, Kocher MS. Differentiating between methicillin-resistant and methicillin-sensitive Staphylococcus aureus osteomyelitis in children: an evidence-based clinical prediction algorithm. J Bone Joint Surg Am. 2011;93:1693-701. 